- Serves as a reminder of a rare complication.

- Outlines points to prevent such a complication.

- Describes current management.

- Illustrates the dentist's role following the event.

\title{
Needle breakage following inferior alveolar nerve block: implications and management
}

\author{
M. Ethunandan, ${ }^{1}$ A. L. $\operatorname{Tran}_{1}{ }^{2}$ R. Anand, ${ }^{3}$ J. Bowden, ${ }^{4}$ M. T. Seal ${ }^{5}$ and P. A. Brennan ${ }^{6}$
}

Needle breakage following inferior alveolar nerve block is a rare complication in current dental practice. We report a case of delayed retrieval of a broken needle with the use of modern imaging modalities. In addition, possible causes of needle breakage and preventative measures, indications and timing of removal, localisation techniques and surgical approaches are discussed.

\section{INTRODUCTION}

The current use of disposable needles made of modern alloys has significantly decreased the incidence of needle breakage during administration of local anaesthesia for dental treatment. ${ }^{1-3}$ This complication, however, continues to be reported infrequently and is thought to be due to poor practice, unexpected patient movement and needle manufacturing defects. ${ }^{1-8}$ Needle breakage has most frequently been reported in relation to an inferior alveolar nerve block and the literature has mainly concentrated on elaborate needle localisation techniques. ${ }^{1-8}$ We report the management of a patient with a broken needle in the pterygomandibular space and discuss the possible causes and preventative measures, the indications and timing of removal, modern modalities of

\footnotetext{
${ }^{1+}$ Specialist Registrar, ${ }^{2}$ Senior House Officer, ${ }^{3}$ Specialist Registrar, ${ }^{4}$ Specialist Registrar, ${ }^{5}$ Consultant, ${ }^{6}$ Professor of Surgery/Consultant, Oral \& Maxillofacial Surgery, Queen Alexandra Hospital, Portsmouth, P06 3LY ${ }^{*}$ Correspondence to: Mr Madanagopalan Ethunandan Email:mgethu@hotmail.com
}

localisation and a technique for needle retrieval. We also give advice for appropriate referral and documentation.

\section{CASE REPORT}

A 45-year-old male was referred by his dentist to the maxillofacial department for management of a broken 25 $\mathrm{mm}$, 30-gauge dental needle in the right pterygomandibular space. This occurred following the administration of a right inferior alveolar nerve block, for the removal of the lower right second molar tooth. The needle had fractured at the hub and was not clinically visible or palpable. On presentation, the patient had no pain, dysphagia or right inferior alveolar nerve paraesthesia.

Dental panoramic, postero-anterior mandible and lateral cephalometric radiographs were taken which confirmed the presence of the needle in the pterygomandibular space (Fig. 1). A computed tomogram (Siemens Somatom Volume Zoom 4), with axial (Fig. 2) and reformatted 3D volume rendered (Fig. 3) images was also obtained which demonstrated the needle to be present immediately adjacent to the medial aspect of the ramus of the mandible and just below the lingula.
In view of the lack of significant symptoms, the patient did not want intervention, but understood the possible risk of needle migration if left in situ. Six months later, the patient complained of persistent discomfort in the mouth and therefore requested removal of the needle. A further dental panoramic radiograph taken at this time did not demonstrate any significant alteration in needle position.

At operation under general anaesthesia, a vertical incision was made along the ascending ramus, which extended to the external oblique ridge, allowing an avascular subperiosteal plane to be established in the medial aspect of the mandible. The lingula, lingual and inferior dental nerves were visualised and protected. Following careful blunt dissection through the periosteum, the broken needle was identified supra-periosteally in the same location as demonstrated in the CT scan and retrieved. The wound was closed using resorbable sutures. The patient made an uneventful recovery.

\section{DISCUSSION}

Needle breakage during administration of an inferior alveolar nerve block is a rare event in modern dental practice and 


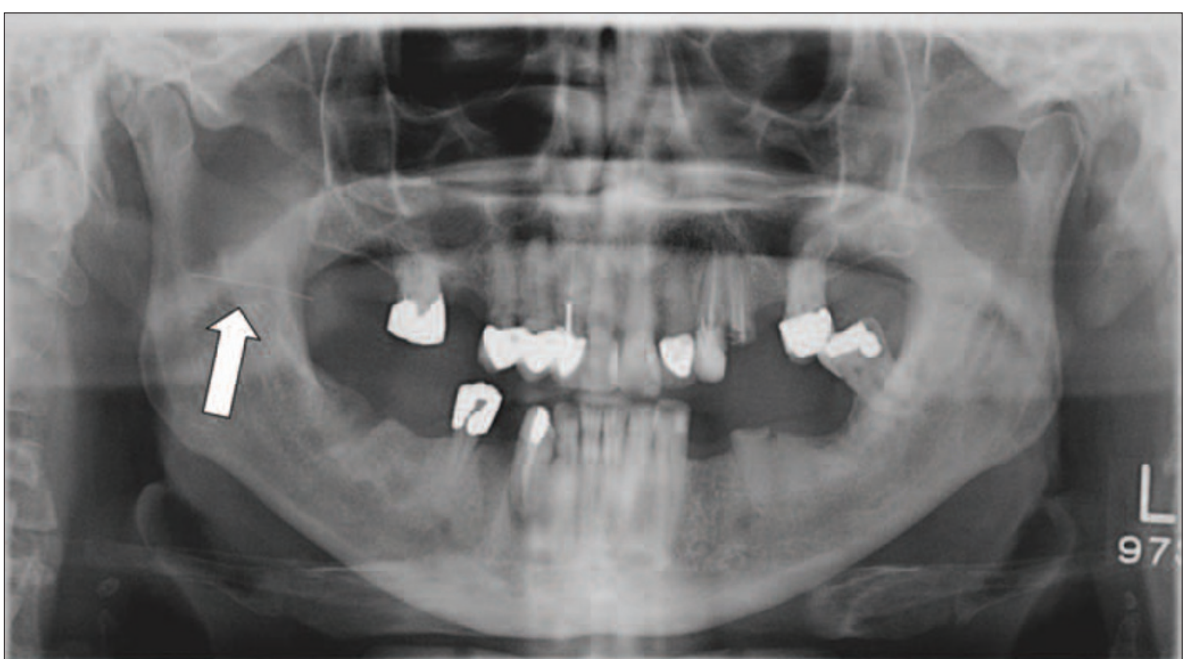

Fig. 1 A dental panoramic radiograph demonstrating the position of the broken needle (arrow)

this reduction is largely due to singleuse disposable needles made of improved stainless steel alloys and conscientious local anaesthetic techniques. ${ }^{1-3}$ When it does happen, it is often a stressful event for the patient and dentist alike. This complication was, however, more frequent prior to the 1960 s as evidenced by Blum's report of 100 cases in $1928^{9}$ and Fraiser-Modie's review of 26 cases in $1958 .{ }^{10}$ This was thought to be at least partly due to the use of more rigid, non disposable needles which were subjected to repeated sterilisation cycles, with attendant alterations to their physical properties during this time. Subsequent reports have often documented isolated cases and in most instances in relation to the use of a 30 gauge needle. ${ }^{1-6}$

It is well recognised that needle breakages are more common with a smaller diameter needle and often occur at the hub. ${ }^{1-8}$ Though most dentists use a 27 gauge $35 \mathrm{~mm}$ (long) needle for administration of an inferior alveolar nerve block in an adult, there is occasionally a perception that the use of a thinner needle (30 gauge) is associated with less discomfort. It has however, been shown that there is little difference in the pain perception between the use of 27 and 30 gauge needles. ${ }^{11}$ It is also known that the deflection of the needle and the pressure exerted on the syringe is greater with a smaller gauge needle. ${ }^{12}$ In addition, bending the needle weakens it as can any significant changes in direction of the needle located deep in the tissues. Finally, a 30 gauge needle often has to be inserted to the hilt to deposit the local anaesthetic in the appropriate position. All of the above can contribute to needle breakage and therefore the use of a 30 gauge needle should be avoided when administering an inferior alveolar nerve block (Table 1).

In the event that needle breakage does occur, every effort should be made to retrieve the needle immediately, if the tip is visible, using fine haemostats. In the unfortunate event of the tip not being visible, the patient should be informed and arrangements should be made for appropriate referral to the maxillofacial unit. If possible, the dentist should speak directly to a senior member of the local maxillofacial unit while the also seen

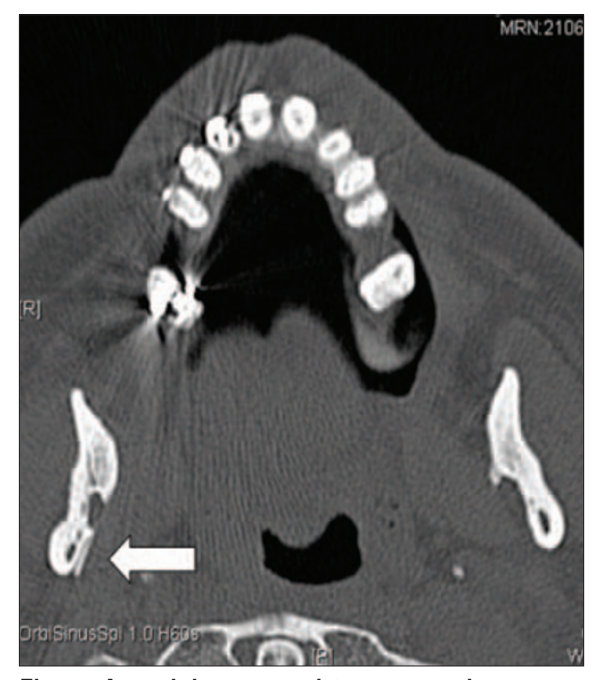

Fig. 2 An axial computed tomogram demonstrating the relationship of the needle (arrow) to the lingula and medial aspect of the ramus

patient is still present to arrange an urgent assessment in addition to sending a referral letter. The remaining portion of the broken needle should also be sent along for further determinations of the size of the broken fragment. This often reassures the patient and the dentist and reinforces the quality of care being provided (Table 2).

Prompt retrieval is advocated to minimise symptoms of pain, dysphagia, trismus and to prevent migration of the needle and potential damage to vital

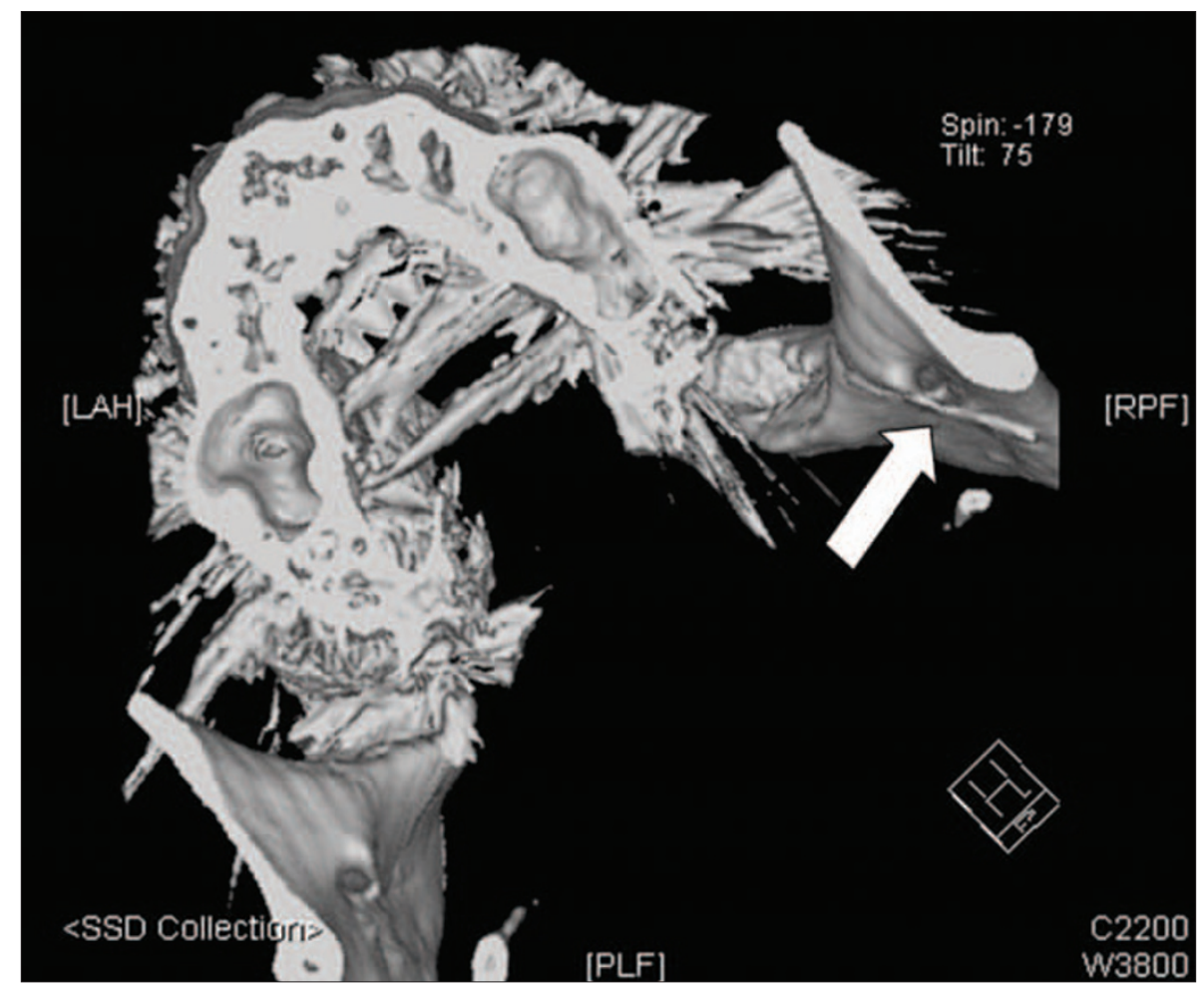

Fig. 3 A reformatted 3D CT scan demonstrating the relationship of the needle (arrow) to the lingula and medial aspect of the ramus. Beam hardening artefacts from the dental restorations are 
structure. ${ }^{1-10}$ The psychological effects of having a sharp object embedded in the mouth should also be considered. ${ }^{1}$ However, retrieval of the needle in itself can lead to neurological and tissue damage during removal and some suggest that removal only be considered if the patient develops symptoms. ${ }^{3}$ Finally, the potential medicolegal implication of the incident needs to be taken into account, though it should not be a primary consideration in the management. In our patient, the initial decision was to leave to needle in situ, but it was subsequently removed six months later due to persisting local symptoms. There was, however, no evidence of needle migration in the intervening period, as demonstrated by the immediate pre-operative radiographs and its presence in its original location in the pterygomandibular space at retrieval (Table 3).

A variety of methods have been described to locate a broken needle in the pterygomandibular space. Plain radiographs taken at right angles to each other are often the initial investigation of choice. The most frequent combinations employed are a dental panoramic radiograph and postero-anterior view of the mandible, though others have been described.1-10 Plain radiographs are useful in confirming the presence, dimensions and approximate position of the needle. They are, however, unable to provide the accurate position of the needle and its relationship to adjacent structures. This information, which may be obtained from a CT scan is invaluable prior to surgical exploration. The incision and site of exploration can be determined from the information available on the CT scan, as demonstrated in our case. We think a CT scan should be the imaging investigation of choice, as it accurately relays the position of the needle to recognisable anatomical landmarks, especially with 3D reformatting (Fig. $3)$. The availability of modern helical CT scanners allows quick acquisition of thinly collimated volumetric data, with slice thickness of $0.5-1 \mathrm{~mm}$, which can subsequently be reformatted to provide detailed 3D images. The presence of dental restorations, however, can occasionally result in beam hardening artefacts which can interfere with the quality of the images obtained.

Various other intra-operative localisation techniques have been described, including the use of intra-operative radiographs with and without preformed stents, metal detectors, ultrasound probes, magnets and image intensifiers. ${ }^{7,8,13-15}$ The use of magnets is no longer suitable, due to the changes in the manufacturing process, where ferrous compounds have been replaced with other alloys. ${ }^{3,13}$ Intra-operative Xrays with positioning stents are often time consuming and the discrimination between small changes in position between the two (three) localising needles can often be poor. ${ }^{7}$ Metal detectors and ultrasound probes are often cumbersome to use intra-orally..$^{14,15}$ Thompson et al. have recently reported the use of an image intensifier, and feel it is a readily available and useful adjunct in the intra-operative localisation of a broken needle. $^{8}$

Most reports have suggested the use of a vertical mucosal incision often on the medial aspect of the mandible in the area penetrated by the needle, followed by blunt supra-periosteal dissection to identify to identify the needle. ${ }^{1-5}$ In our case, we employed a vertical incision along the anterior border of the mandible, extending inferiorly to the external oblique ridge, followed by subperiosteal dissection along the medial aspect of the ramus of the mandible. We feel the initial subperiosteal dissection help identify bony landmarks (lingula), which can be used as reference during exploration and also provides greater protection to the inferior alveolar and lingual nerves. Focussed extra periosteal dissection, based on CT scan information can be subsequently carried out without inadvertent damage to these nerves.

Finally, the maintenance of accurate, contemporaneous notes cannot be overstressed. The patient should be fully informed of the events and arrangements made for referral to the maxillofacial unit. It is also prudent to inform and seek advice from the dental/medical insurance provider.

1. Marks R B, Carlton D M, McDonald S. Management of a broken needle in the pterygomandibular space: report of a case. JADA 1984; 109: 263-264.

2. Bedrock R D, Skigen A, Franklin-Dolwick M. Retrieval of a broken needle in the pterygomandibular space. JADA 1999; 130: 685-687.

3. Faura-Solé M, Sánchez-Garcés M A, Berini-Aytes L et al. Broken anaesthetic injection needles: report of 5 cases. Quint Int 1999; 30: 461-465.

4. Bhatia S, Bounds G. A broken needle in the pterygomandibular space: report of a case and review of the literature. Dent Update 1998; 25: 35-37.

\begin{tabular}{l} 
Table 1 Prevention \\
\hline Use 27 gauge long needle \\
\hline Avoid bending needle \\
\hline Avoid inserting needle to its hilt \\
Avoid major changes in position of the needle \\
during administration
\end{tabular}

\begin{tabular}{l} 
Table 2 Management by the dentist \\
\begin{tabular}{l|l} 
If tip visible & $\begin{array}{l}\text { Attempt removal with a fine } \\
\text { haemostat }\end{array}$ \\
\hline If tip not visible & $\begin{array}{l}\text { Inform patient and avoid } \\
\text { excessive jaw movements } \\
\text { to maxillofacial unit }\end{array}$ \\
Maintain contemporaneous \\
records \\
Inform your defence union
\end{tabular} \\
\hline
\end{tabular}

Table 3 Management by maxillofacial surgeon

Arrange immediate assessment of patient

Assess needle position (X-rays, CT scan)

Discuss treatment options (conservative) immediate or delayed retrieval)

Organise surgical exploration/follow up

5. Zelter $\mathrm{R}$, Cohen $\mathrm{C}$, Casap N. The implications of a broken needle in the pterygomandibular space: clinical guidelines for prevention and retrieval. Paediatric Dent 2002: 24: 153-156.

6. McDonough T. An unusual case of trismus and dysphagia. Br Dent J 1996; 180: 465-466.

7. Ho K H. A simple technique for localising a broken dental needle in the pterygomandibular region. Aust Dent J 1988; 33: 308-309.

8. Thompson M, Wright S, Cheng L H H et al. Locating broken dental needles. Int J Oral Maxillofac Surg 2003; 32: 642-644.

9. Blum T. A report of 100 cases of hypodermic needles broken during the administration of oral local anaesthesia. Dent Cosmos 1928; 70: 865-874.

10. Fraser-Moodie W. Recover of broken needles. BrDent J 1958; 105: 79-85.

11. Fuller N P, Menke R A, Meyers W J. Perception of pain to three different intra-oral penetration of needles. J Am Dent Assoc 1979; 99: 822-824.

12. Aldons J A. Needle deflection: a factor in the administration of local anaesthetics. J Am Dent Assoc 1968; 77: 602-604.

13. Johansson B, Krekmanov L. Fragment of broken instrument removed from field of operation by an electromagnet. Br J Oral Maxillofac Surg 1987; 25: 265-266.

14. Moore U J, Fanibunda K, Gross M J. The use of a metal detector for localisation of a metallic foreign body in the floor of the mouth. Br J Oral Maxillofac Surg 1993; 31: 191-192.

15. Abe K, Nakamatsu K, Beppu K et al. Use of intraoperative ultrasonography to detect a small foreign body in the soft tissues of the upper lip. BrDent J 1994; 177: 292-294. 